\title{
Elective hip arthroplasty rates and related complications in people with diabetes mellitus
}

HIP International

2022, Vol. 32(6) 717-723

(C) The Author(s) 2020

\section{(c) (i) (5)}

Article reuse guidelines: sagepub.com/journals-permissions DOI: | 0.1 | 777/ | | 2070002098| 573 journals.sagepub.com/home/hpi

(S)AGE

\author{
Lindsey C McVey' ${ }^{\mathbb{D}}$, Nicholas Kane ${ }^{2}$, Helen Murray², \\ RM Dominic Meek ${ }^{2}$ and S Faisal Ahmed'
}

\begin{abstract}
Background and Aims: Diabetes mellitus (DM), poor glycaemic control and raised body mass index (BMI) have been associated with postoperative complications in arthroplasty, although the relative importance of these factors is unclear. We describe the prevalence of DM in elective hip arthroplasty in a UK centre, and evaluate the impact of these factors. Methods: We analysed retrospective data for DM patients undergoing arthroplasty over a 6-year period and compared with non-diabetic matched controls (I DM patient: 5 controls). DM was present in $5.7 \%$ of hip arthroplasty patients (82/l443).

Results: Postoperative complications occurred in $12.2 \%$ of DM patients versus $12.9 \%$ of controls $(p=1.000)$; surgical complications were present in $6.1 \%$ of those with DM and $2.4 \%$ of controls $(p=0.087)$, while medical complications occurred in $8.5 \%$ of DM patients versus $10.7 \%$ of controls $(p=0.692)$. Complications developed in $23.1 \%$ of DM patients with poor glycaemic control $(\mathrm{HbAlc}>53 \mathrm{mmol} / \mathrm{mol})$ versus $9.8 \%$ with good control $(p=0.169)$. In DM patients and controls combined, complications occurred in $16.3 \%$ of obese patients versus $10.0 \%$ of non-obese patients $(p=0.043)$. In the DM cohort, I3.7\% of overweight patients had complications versus $0 \%$ with a normal or low BMI ( $p=0.587)$.

Conclusions: DM rates were lower than expected, and glycaemic control was good. Overall complication rates were unrelated to the presence of DM or to glycaemic control, although surgical complications were observed more frequently in those with DM and poor glycaemic control was uncommon within our cohort. Complications were more frequent in those with a higher BMI. Whether some patients with DM but without an increased risk of complications are currently being excluded from surgery requires exploration.
\end{abstract}

Keywords

Blood Glucose, body mass index, glycated haemoglobin A, obesity, osteoarthritis

Date received: 8 November 2019; accepted: 24 November 2020

\section{Introduction}

Osteoarthritis is the commonest joint disease worldwide. It is a highly disabling condition, with a large burden of pain, loss of function and socioeconomic cost. ${ }^{1}$ The major risk factors for osteoarthritis are obesity and ageing., ${ }^{2,3}$ Excessive weight increases joint loading, resulting in cartilage breakdown and joint remodelling; while ageing reduces the capacity of articular cartilage to adapt to mechanical stress, causing catabolism and cell death. ${ }^{3,4}$

Diabetes mellitus (DM) is also a common condition in obesity and advanced age..$^{5-7}$ It is therefore unsurprising that DM and osteoarthritis are often present in the same patient. Hyperglycaemia also contributes directly to the cartilage damage seen in OA; via oxidative stress, proinflammatory pathways and stimulation of bone remodelling,

\footnotetext{
'Developmental Endocrinology Research Group, School of Medicine, University of Glasgow, Glasgow, UK

${ }^{2}$ Department of Trauma and Orthopaedics, Queen Elizabeth University Hospital, Glasgow, UK

Corresponding author:

S Faisal Ahmed, Developmental Endocrinology Research Group, School of Medicine, Dentistry and Nursing, University of Glasgow, Royal Hospital for Children, I345 Govan Road, Glasgow, G5I 4TF, UK. Email: faisal.ahmed@glasgow.ac.uk
} 
further strengthening the links between these conditions. ${ }^{8}$ $\mathrm{DM}$ and osteoarthritis are becoming increasingly common - mainly due to the ageing global population and escalating obesity crisis - meaning that increasing numbers suffer from both conditions. ${ }^{1,5}$

The overwhelming majority of the 90,000 primary elective hip arthroplasties performed in the UK each year are for osteoarthritis. ${ }^{9}, 10$ It follows that we have a large arthroplasty population at high risk of DM. In the USA, around $9.3 \%$ of the adult population have $\mathrm{DM},{ }^{11}$ while the rate in elective arthroplasty patients is almost double this value. ${ }^{12,13}$ Although the rate of DM in the UK adult population is lower $(6.2 \%)$, the prevalence in UK arthroplasty patients is less clear. ${ }^{14}$

$\mathrm{DM}$ is associated with postoperative complications in lower limb arthroplasty, including prosthetic joint infections, increased hospital costs, and persistent pain. ${ }^{13,15,16}$ When diabetes is poorly controlled, postoperative morbidity and mortality may be greater still. ${ }^{17,18}$ Given that only $1 / 4$ of UK diabetic patients have good glycaemic control, this presents a heavy burden in healthcare costs. ${ }^{19}$ Some centres in the USA now risk-stratify elective arthroplasty patients and redirect those with poorly-controlled diabetes to primary care services, until their $\mathrm{HbA} 1 \mathrm{c}$ is $<53 \mathrm{mmol} / \mathrm{mol}^{20}$ Although this approach should make surgery safer, it is controversial as it delays joint arthroplasty procedures for up to $1 / 3$ of patients, a significant proportion fail to achieve improved diabetic control, and evidence of the best threshold value for $\mathrm{HbA1c}$ is inconsistent. ${ }^{17,20-22}$

Increased body mass index (BMI) is a well-studied risk factor for complications following arthroplasty, including prosthetic joint infection and cardiac complications. ${ }^{23,24}$ In a recent meta-analysis, increased BMI correlated with surgical site infection in a dose-response relationship. ${ }^{25}$ Obesity is extremely common in patients with type $2 \mathrm{DM}$ and accounts for $>80 \%$ of the risk of developing this disease. ${ }^{26}$ Increased BMI is likely to be a common and important risk factor for complications in patients with DM undergoing joint arthroplasty, although the relative importance of obesity and poor glycaemic control is less clear.

The purpose of this study was, firstly, to describe the prevalence of DM in patients undergoing elective hip arthroplasty in a large Scottish centre; secondly, to compare the postoperative complication rates in these cases to non-diabetic age- and sex-matched controls; and lastly to investigate the relationship between postoperative complications and preoperative glycaemic control and BMI.

\section{Methods}

This was a retrospective study of patients with DM undergoing elective total hip arthroplasty (THA) at a large Scottish teaching hospital from January 2009 until May 2015.

\section{Sampling criteria and data sources}

A local database was used to identify all elective THA procedures undertaken at the site. Revision hip arthroplasty was excluded as this is associated with higher complication rates. ${ }^{27}$ Patients were then linked to Scottish Care Information (SCI)-diabetes (a comprehensive national information system which supports the care of all patients with DM across Scotland), using their Community Health Index (CHI) number, to identify those with DM. ${ }^{28}$ Clinical data from multiple databases were analysed, including a detailed preoperative clinic proforma (see appendix).

\section{Review of patient records}

Demographic information including age, gender, selfreported smoking status and level of alcohol consumption were collected. Postcode was used to look up the patient's Scottish Index of Multiple Deprivation (SIMD) decile from a Scottish Government database. ${ }^{29}$ This reflects the level of deprivation in the patient's home community $(1=$ most deprived decile, $10=$ least deprived $)$. American Society of Anesthesiologists (ASA) score was recorded from the anaesthetic chart. Diabetic medication was recorded, and patients were categorised as 'diet controlled,' 'antidiabetic medication controlled' or 'insulin controlled.' Procedure variables were also collected. Preoperative HbA1c (a measure of glycaemic control reflecting the 3 months prior to sampling), as measured in the year prior to the arthroplasty procedure, was recorded. If multiple results were available, the $\mathrm{HbA} 1 \mathrm{c}$ value closest to the date of surgery was used. BMI was classed as overweight at $25.0 \mathrm{~kg} / \mathrm{m}^{2}$ or greater, and obese at $\geqslant 30.0 \mathrm{~kg} /$ $\mathrm{m}^{2}$. Electronic case notes pertaining to the patient's admission, any subsequent admissions and clinic appointments to 1-year post-arthroplasty were reviewed by an orthopaedic research nurse and postoperative complications were documented.

\section{Control patients}

For each patient with DM, 5 age- and sex-matched nondiabetic controls were identified, who had undergone primary elective hip arthroplasty at the same centre. Demographic and procedure variables were collected, and the rate of postoperative complications was analysed.

\section{Statistical analysis}

Data were compared using the Student's $t$-test or Fisher's exact test as appropriate. Bonferroni correction was applied firstly to the set of hypothesis tests for demographic data and then to the tests for postoperative complications to reduce type I error. Statistical significance was set at $p<0.05$. 
Table I. Type of postoperative complication and frequency in patients with diabetes mellitus (DM) and controls.

\begin{tabular}{|c|c|c|c|c|}
\hline \multirow[t]{2}{*}{ Complication } & \multicolumn{2}{|c|}{ DM patients $(n=82)$} & \multicolumn{2}{|c|}{ Controls $(n=4 \mid 0)$} \\
\hline & Number & Percentage & Number & Percentage \\
\hline Deep wound infection & 4 & $4.9 \%$ & 3 & $0.7 \%$ \\
\hline Postoperative loosening & 0 & - & 2 & $0.5 \%$ \\
\hline Postoperative dislocation & 2 & $2.4 \%$ & 5 & $1.2 \%$ \\
\hline Total number of patients with surgical complications & 5 & $6.1 \%$ & 10 & $2.4 \%$ \\
\hline Superficial wound infection & 1 & $1.2 \%$ & 7 & $1.7 \%$ \\
\hline Acute kidney injury & 3 & $3.7 \%$ & 6 & $1.5 \%$ \\
\hline Chest or urine infection & 1 & $1.2 \%$ & 12 & $2.9 \%$ \\
\hline Stroke & 1 & $1.2 \%$ & 1 & $0.2 \%$ \\
\hline Hypotension requiring resuscitation or critical care admission & 0 & - & 5 & $1.2 \%$ \\
\hline Pulmonary embolism or deep vein thrombosis & 0 & - & 9 & $2.2 \%$ \\
\hline Haematemesis & 0 & - & 3 & $0.7 \%$ \\
\hline Congestive cardiac failure & 0 & - & 1 & $0.2 \%$ \\
\hline Other & 1 & $1.2 \%$ & 5 & $1.2 \%$ \\
\hline Total number of patients with medical complications & 7 & $8.5 \%$ & 44 & $10.7 \%$ \\
\hline Total number of patients with complications & 10 & $12.2 \%$ & 53 & $12.9 \%$ \\
\hline
\end{tabular}

Note: Multiple complications were present in 3 patients with DM (3.7\%) and in 6 controls (I.5\%).

\section{Ethics}

This study was a retrospective case sheet-based analysis only. Approval was granted by the clinical director for orthopaedics.

\section{Results}

\section{DM rate and demographics}

During the study period 1443 patients underwent elective primary THA, performed by a team of 10 consultant orthopaedic surgeons. Of these patients, $82(5.7 \%)$ had a diagnosis of DM. We studied 410 age- and sex-matched non-diabetic controls.

Within the diabetic cohort, $50(61.0 \%)$ patients were female and median age was 71 years (interquartile range (IQR) 64:75 years). Most had type $2 \mathrm{DM}(97.6 \%)$ and type 1 DM was present in only 2 patients. Diabetes was managed by diet alone in 30 patients $(36.6 \%)$, oral antidiabetic drugs in $46(56.1 \%)$, insulin monotherapy in $4(4.9 \%)$, and insulin in addition to oral antidiabetic agents in $2(2.4 \%)$.

Smoking was more common in patients with DM (current smokers 15 [18.3\%] DM vs. 36 (8.9\%) controls; $p=0.017)$; and excess alcohol consumption was similar (7 (8.5\%) DM vs. 59 [14.6\%] controls; $p=0.161)$. SIMD decile was lower in patients with DM (median 4 [IQR $2: 8])$; than controls (6 [3:9]; $p=0.015)$, indicating higher levels of deprivation; and ASA score was higher (DM median 2 [IQR 2:3]; controls 2 [2:2]; $p<0.001$ ), indicating greater co-morbidity. Anaesthetic information was available for 67 patients with DM (general anaesthesia [GA] 23 [34.3\%], spinal block 42 [62.7\%], both 2 [3.0\%]) and 393 controls (GA 107 [27.2\%], spinal block 255 [64.9\%], both 31 [7.9\%]).
HbA1c was measured in the year prior to surgery in 67 $(81.7 \%)$ patients with DM with a median of $49 \mathrm{mmol} / \mathrm{mol}$ (IQR 43:62). HbAlc was $>53 \mathrm{mmol} / \mathrm{mol}$ in 26 patients $(38.8 \%)$, and $>58 \mathrm{mmol} / \mathrm{mol}$ in 18 patients $(26.9 \%)$. BMI was greater in patients with DM than controls (DM median 32 [IQR 27:36]; controls 29 [26:33]; $p=0.003$ ).

\section{Postoperative complications: impact of DM}

Postoperative complications were equally likely patients with DM (10 patients, 12.2\%) and controls (53 patients, $12.9 \%)(p=1.000)$. Complications requiring surgical intervention occurred in $5 \mathrm{DM}$ patients $(6.1 \%)$ and 10 controls $(2.4 \%)(p=0.087)$; while medical complications were present in 7 DM patients $(8.5 \%)$ and 44 controls $(10.7 \%)$ $(p=0.692)$. Details of specific postoperative complications are displayed in Table 1.

\section{Postoperative complications: impact of glycaemic control (illustrated in Figure I(A))}

The risk of complications in those with preoperative $\mathrm{HbA} 1 \mathrm{c}>53 \mathrm{mmol} / \mathrm{mol}$ was $23.1 \%$ (6/26 patients with $\mathrm{HbA} 1 \mathrm{c}>53)$ versus $9.8 \%(4 / 41)$ in those with $\mathrm{HbA} 1 \mathrm{c} \leqslant 53$ $(p=0.169)$; the corresponding figures for those with $\mathrm{HbA} 1 \mathrm{c}>58 \mathrm{mmol} / \mathrm{mol}$ was $16.7 \%(3 / 18)$ versus $14.3 \%$ (7/49) with $\mathrm{HbA} 1 \mathrm{c} \leqslant 58(p=1.000)$.

\section{Postoperative complications: impact of BMI (illustrated in Figure I(B) and (C))}

Risk of complications in overweight patients (DM and control patients combined) was $13.4 \%$ (54/403 overweight 


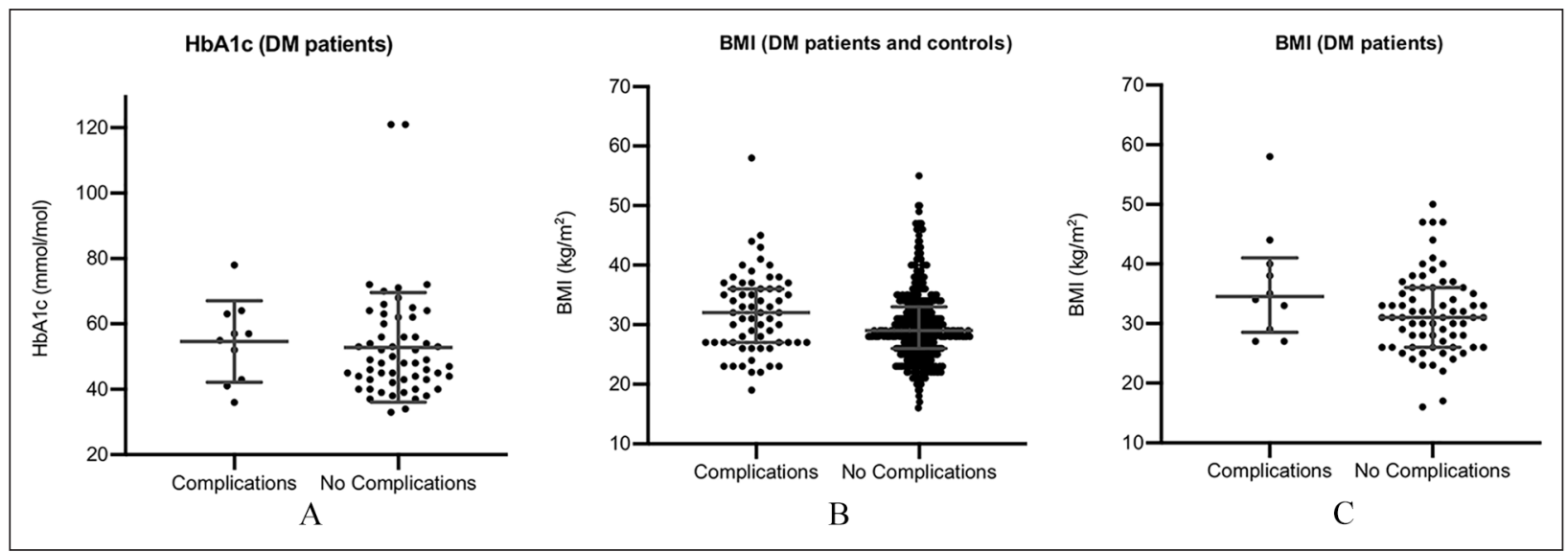

Figure I. The graphs compare characteristics of uncomplicated arthroplasty versus those procedures with postoperative complications (A) HbAlc (DM patients, $n=67$ ) (B) BMI (DM and controls, $n=483$ ) (C) BMI (DM patients, $n=80$ ).

patients) versus $11.3 \%(9 / 80)$ in those with a normal weight $(p=0.718)$; while risk in obese patients was $16.3 \%$ $(38 / 233)$ versus $10.0 \%(25 / 250)$ in non-obese patients $(p=0.043)$. The corresponding risks within the DM cohort were $13.7 \%(10 / 73)$ in overweight patients versus $0 \%$ $(0 / 7)$ in normal weight $(p=0.587) ; 13.7 \%(7 / 51)$ in obese patients versus $10.3 \%(3 / 29)$ in non-obese $(p=0.740)$.

\section{Discussion}

In a large Scottish teaching hospital, $5.7 \%$ of patients undergoing elective hip arthroplasty had DM. This is similar to the population rate of DM $(6.2 \%$ in the UK, $5.4 \%$ locally in Scotland), but lower than expected in patients with osteoarthritis (with relatively advanced age and high $\mathrm{BMI}$ ), and certainly lower than rates reported previously in North America. ${ }^{12,13,30}$

Our arthroplasty patients with DM enjoyed relatively good health and tight glycaemic control. We would expect that the ASA score (an indicator of pre-existing morbidity) would be higher in a patient with DM than in an unaffected arthroplasty patient. If DM was associated with complications such as cardiovascular or renal disease, the ASA score might be higher still. Despite this, scores were comparable between our cohorts, indicating low levels of comorbidity in our DM arthroplasty patients. Glycaemic control was poor in less than $40 \%$ (HbA1c $>53 \mathrm{mmol} /$ mol), which is much lower than estimated rates in the background population (75\%; Diabetes UK). ${ }^{19}$

We argue that an assessment of the patient's overall health - including the presence of DM and related complications - forms an important part of our operative selection process, resulting in fewer patients with DM undergoing elective arthroplasty. This selection process takes place at multiple levels - the patient is perhaps less likely to request surgery when their health is poor, the General Practitioner may be less likely to refer a high-risk patient to clinic, and the Orthopaedic Surgeon or Anaesthetist will counsel against arthroplasty where the operative risk outweighs the potential gains in function. As a unit, we do not apply any formal cut-offs (for example for HbA1c or BMI), although an assessment of which values make surgery less likely to progress would be an interesting avenue for future research.

Our control group was individually matched for gender and age. Patients with DM were more likely to smoke and to belong to a lower socio-economic group, although there was no difference in excess alcohol consumption. These demographics may be reflective of the Scottish DM arthroplasty population at large - further research here may be helpful. The discrepancy in BMI between the 2 groups was expected, given the link between obesity and DM. The type of anaesthetic used was also similar.

Postoperative complication rates were similar in DM patients and controls, while differences were observed in the commoner types of complications in each group. Notably, deep wound infection and postoperative dislocation were commoner in the DM group, although these were observed differences only, as event numbers were not large enough to allow statistical testing. These complications are particularly serious, as they result in repeated orthopaedic procedures, with repeated risks of postoperative complications. Observed rates of venous thromboembolism were lower in DM patients than controls, perhaps related to the more aggressive deep vein thrombosis (DVT) prophylaxis regimen implemented locally in patients with a greater BMI. ${ }^{31}$ It is possible that this more aggressive regimen may lead to increased postoperative infection rates, via increased haematoma formation, as has been observed in a previous study. ${ }^{32}$ Rates of medical complications were similar in patients with DM and controls, except for rates of acute kidney injury, which were around twice as common in the DM group - this was to be expected given the renal complications associated with DM. 
Contrary to some studies, we did not find any difference in postoperative complication rates in those with a higher $\mathrm{HbAlc}$, although the good glycaemic control seen in most of our patients resulted in limited numbers with a high $\mathrm{HbAlc}$. In a retrospective study of over 1 million arthroplasty patients in the USA, those with uncontrolled DM had a higher likelihood of complications, including mortality, ${ }^{18}$ although the effect size was small in this very large study and thus clinical significance is less certain. More recent large studies have failed to show a correlation between $\mathrm{HbAlc}$ and complications, ${ }^{13,17}$ and a recent systematic review concluded that $\mathrm{HbAl} \mathrm{c}$ was not associated with postoperative morbidity (including surgical site infection) or mortality. ${ }^{33}$ The impact of glycaemic control on postoperative complications therefore remains unclear. It could, therefore, be worth exploring whether some patients with poorer glycaemic control who are not currently undergoing elective arthroplasty procedures could receive surgery without an unacceptably higher risk of complications.

Our finding of a higher risk of postoperative complications in obese patients was in keeping with previous studies. ${ }^{34}$ The risk of complications in arthroplasty rises as BMI increases, especially when BMI is $>50 \mathrm{~kg} / \mathrm{m}^{2} .25,35$ Rates of surgical site infection in particular are higher in obesity - this finding was reproduced in a recent US cohort of hip arthroplasty patients with a BMI of greater than $40 \mathrm{~kg} / \mathrm{m}^{2} .{ }^{24,36}$ We note that overall complication rates in this study were lower than in our patients. This is likely to be related to the shorter follow-up period (30 days compared with 1 year in our study) and exclusion of acute kidney injury as a recognised postoperative complication in the US study. We report increased complications in obese patients (BMI $>30 \mathrm{~kg} / \mathrm{m}^{2}$ ) in our mixed cohort (including patients with DM and non-diabetic controls). In our DM patients, risk appears to be elevated at a lower BMI cut-off $\left(>25 \mathrm{~kg} / \mathrm{m}^{2}\right)$, although the lack of events in DM patients with a normal weight means that our statistical analysis is likely to be under-powered.

Our study shows that postoperative complications are more common in those patients with a higher BMI. We argue that this could be an effective target for reducing surgical risk in all patients, including those with DM, although weight reduction is often challenging. With the limitation of our low event numbers, our data suggest that a BMI target of $30 \mathrm{~kg} / \mathrm{m}^{2}$ may be appropriate. This target requires evaluation in larger studies.

Within our cohort, a recent $\mathrm{HbA} 1 \mathrm{c}$ test (within 1 year preoperatively) was not performed in nearly $1 / 5$ of our patients. In this group, 2 patients had postoperative complications $(13.3 \%)$, which is similar to our other groups. Annual HbA1c checks are routinely provided at diabetes check-ups, and so patients without an HbA1c check in the year preceding arthroplasty may be classed as clinic nonattenders and are more likely to have poor glycaemic control. ${ }^{37,38}$ Although we have not shown an effect on outcomes in arthroplasty, the high proportion of patients not receiving routine checks requires further attention, as those with poor control are undoubtedly at greater risk of microvascular and cardiovascular complications of DM. We suggest that efforts should be made at every healthcare encounter, including at preoperative assessment and elective surgery, to refer these patients to diabetes services in primary or secondary care.

A major strength of this study was its universal approach; we included all patients with DM operated upon at the centre within our time frame. In Scotland, we benefit from excellent databases - over $99 \%$ of our patients with DM are registered on both clinical and research databases - and so we expect that our data are inclusive. ${ }^{39} \mathrm{We}$ analysed clinical data from a number of electronic sources (including scanned copies of medical notes), providing us with comprehensive information on our patients. Given the retrospective design of this study, some data were missing, including $\mathrm{HbA} 1 \mathrm{c}$ values for nearly $1 / 5$ of our patients with DM. While this precludes analysis on glycaemic control in these patients (which may be poor), this is an accurate reflection of the information available in our current clinical practice, which we believe is typical of UK practice. Our DM group tended to have good glycaemic control, which limited our analysis of the impact of HbAlc on complication rates. Although this provides a clear illustration of our practice, it may also be interesting to analyse complication rates in a cohort with poorer control. Complication rates in a larger cohort of DM patients with a normal weight could also be explored, although given the very high prevalence of overweight and obesity in DM patients requiring arthroplasty, recruitment may be challenging.

In conclusion, in our cohort of patients selected for elective hip arthroplasty, the rate of DM was similar to the rate in the background population, and lower than might be expected in patients with osteoarthritis. Glycaemic control was good, but HbAlc screening was not universal. Postoperative complication rates were similar in DM patients and controls, although surgical complications such as deep wound infection were observed more frequently in the DM group. Complication rates were not influenced by glycaemic control, although poor glycaemic control was uncommon. This suggests that we are selecting good candidates with DM for surgery, which is in turn leading to the low rates of complications. Complications were commoner in patients with a higher BMI, although event numbers were low. The extent to which we may be excluding some patients with DM from arthroplasty who may not be at an increased risk of complications could be a direction for future studies, although rates of surgical complications in this group should be closely monitored.

\section{Acknowledgements}

We thank Dr Brian Kennon and Kevin McGoogan for their assistance with the SCI-diabetes database. 


\section{Declaration of conflicting interests}

The author(s) declared no potential conflicts of interest with respect to the research, authorship, and/or publication of this article.

\section{Funding}

The author(s) received no financial support for the research, authorship and/or publication of this article.

\section{ORCID iD}

Lindsey C McVey (iD https://orcid.org/0000-0003-4756-4092

\section{Supplemental material}

Supplemental material for this article is available online.

\section{References}

1. Hunter DJ and Bierma-Zeinstra S. Osteoarthritis. Lancet 2019; 393: 1745-1759.

2. Anderson JJ and Felson DT. Factors associated with osteoarthritis of the knee in the first national Health and Nutrition Examination Survey (HANES I). Evidence for an association with overweight, race, and physical demands of work. Am J Epidemiol 1988; 128: 179-189.

3. Shane Anderson A and Loeser RF. Why is osteoarthritis an age-related disease? Best Pract Res Clin Rheumatol 2010; 24: 15-26.

4. Kulkarni K, Karssiens T, Kumar V, et al. Obesity and osteoarthritis. Maturitas 2016; 89: 22-28.

5. World Health Organisation. Global report on diabetes. Geneva: World Health Organisation, http://www.who.int/ diabetes/global-report/en/ (accessed 31 March 2018).

6. NCD Risk Factor Collaboration (NCD-RisC). Trends in adult body-mass index in 200 countries from 1975 to 2014: a pooled analysis of 1698 population-based measurement studies with 19.2 million participants. Lancet 2016; 387: 1377-1396.

7. Wild S, Roglic G, Green A, et al. Global prevalence of diabetes: estimates for the year 2000 and projections for 2030 . Diabetes Care 2004; 27: 1047-1053.

8. Zhuo Q, Yang W, Chen J, et al. Metabolic syndrome meets osteoarthritis. Nat Rev Rheumatol 2012; 8: 729-737.

9. NHS National Services Scotland. Scottish arthroplasty project. National report 2017, https://www.arthro.scot.nhs.uk/ Reports/Previous.html (2017, accessed 2 April 2018).

10. National Joint Registry Centre. National Joint Registry for England, Wales, Northern Ireland and the Isle of Man. 14th annual report, National Joint Registry, http://www. njrcentre.org.uk/njrcentre/Reports,PublicationsandMinutes/ Annualreports/tabid/86/Default.aspx (2017, accessed 2 April 2018).

11. National Center for Chronic Disease Prevention and Health Promotion. National diabetes statistics report, https://www. cdc.gov/diabetes/data/statistics/statistics-report.html (2017, accessed 2 April 2018).

12. Capozzi JD, Lepkowsky ER, Callari MM, et al. The prevalence of diabetes mellitus and routine hemoglobin A1c screening in elective joint arthroplasty patients. $J$ Arthroplasty 2017; 32: 304-308.
13. Maradit Kremers H, Lewallen LW, Mabry TM, et al. Diabetes mellitus, hyperglycaemia, haemoglobin A1c and the risk of prosthetic joint infections in total hip and knee arthroplasty. J Arthroplasty 2015; 3: 439-443.

14. Holman N, Young B and Gadsby R. Current prevalence of type 1 and type 2 diabetes in adults and children in the UK. Diabet Med 2015; 32: 1119-1120.

15. Bolognesi MP, Marchant MH Jr, Viens NA, et al. The impact of diabetes on perioperative patient outcomes after total hip and total knee arthroplasty in the United States. $J$ Arthroplasty 2008; 23(Suppl. 1): 92-98.

16. Rajamäki TJ, Jämsen E, Puolakka PA, et al. Diabetes is associated with persistent pain after hip and knee replacement. Acta Orthop 2015; 86: 586-593.

17. Harris AH, Bowe TR, Gupta S, et al. Hemoglobin A1C as a marker for surgical risk in diabetic patients undergoing total joint arthroplasty. J Arthroplasty 2013; 28(Suppl. 8): 25-29.

18. Marchant MH Jr, Viens NA, Cook C, et al. The impact of glycemic control and diabetes mellitus on perioperative outcomes after total joint arthroplasty. J Bone Joint Surg Am 2009; 91: 1621-1629.

19. Diabetes UK. Diabetes: facts and stats, https://www.diabetes.org.uk/Documents/About\%20Us/Statistics/Diabeteskey-stats-guidelines-April2014.pdf (2014, accessed 10 April 2017).

20. Giori NJ, Ellerbe LS, Bowe T, et al. Many diabetic total joint arthroplasty candidates are unable to achieve a preoperative hemoglobin A1c goal of $7 \%$ or less. J Bone Joint Surg Am 2014; 96: 500-504.

21. Shohat N, Muhsen K, Gilat R, et al. Inadequate glycemic control is associated with increased surgical site infection in total joint arthroplasty: a systematic review and metaanalysis. J Arthroplasty 2018; 33: 2312-2321.e3.

22. Yang L, Sun Y, Li G, et al. Is hemoglobin Alc and perioperative hyperglycemia predictive of periprosthetic joint infection following total joint arthroplasty?: a systematic review and meta-analysis. Medicine (Baltimore) 2017; 96: e8805.

23. Gurunathan U, Anderson C, Berry KE, et al. Body mass index and in-hospital postoperative complications following primary total hip arthroplasty. Hip Int 2018; 28: 513621.

24. Toro MD, Peñas C, Abarracín AC, et al. Development and validation of baseline, perioperative and at-discharge predictive models for postsurgical prosthetic joint infection. Clin Microbiol Infect 2019; 25: 196-202.

25. Kunutsor SK, Whitehouse MR, Blom AW, et al. Patientrelated risk factors for periprosthetic joint infection after total joint arthroplasty: a systematic review and meta-analysis. PLoS One 2016; 11: e0150866.

26. Diabetes UK. Diabetes: facts and stats, https://diabetesresources-production.s3-eu-west-1.amazonaws.com/diabetesstorage/migration/pdf/DiabetesUK_Facts_Stats_Oct16. pdf (2016, accessed 10 April 2018).

27. Nishio S, Fukunishi S, Yoshiya S, et al. Comparison of complications following revision of metal-on-metal versus metal-on-polyethylene total hip arthroplasty. Orthopedics 2017; 40: e164-e169.

28. Cunningham S, McAlpine R, Leese G, et al. Using web technology to support population-based diabetes care. $J$ Diabetes Sci Technol 2011; 5: 523-534. 
29. Scottish Government. The Scottish index of multiple deprivation, http://www.gov.scot/Topics/Statistics/SIMD (2017, accessed 26 April 2017).

30. Scottish Diabetes Survey Monitoring Group. Scottish diabetes survey 2016. NHS Scotland, http://www.diabetesinscotland.org.uk/Publications.aspx (accessed 2 April 2018).

31. Healthcare Improvement Scotland $\mid$ Scottish Intercollegiate Guidelines Network. Prevention and management of venous thromboembolism; a national clinical guideline, https://www.sign.ac.uk/sign-122-prevention-and-management-of-venous-thromboembolism.html (2014, accessed 4 September 2018).

32. Agaba P, Kildow BJ, Dhotar H, et al. Comparison of postoperative complications after total hip arthroplasty among patients receiving aspirin, enoxaparin, warfarin and factor Xa inhibitors. J Orthop 2017; 14: 537-543.

33. Rollins KE, Varadhan KK, Dhatariya K, et al. Systematic review of the impact of $\mathrm{HbA1c}$ on outcomes following surgery in patients with diabetes mellitus. Clin Nutr 2016; 35: 308-316.
34. Haynes J, Nam D and Barrack RL. Obesity in total hip arthroplasty: does it make a difference? Bone Joint J 2017; 99-B(Suppl. A): 31-36.

35. Werner BC, Higgins MD, Pehlivan HC, et al. Super obesity is an independent risk factor for complications after primary total hip arthroplasty. J Arthroplasty 2017; 32: 402-406.

36. Foreman CW, Callaghan JJ, Brown TS, et al. Total joint arthroplasty in the morbidly obese: how body mass index $\geqslant 40$ influences patient retention, treatment decisions, and treatment outcomes. J Arthroplasty 2020; 35: 39-44.

37. Diabetes UK. What are the 15 healthcare essentials? https://www.diabetes.org.uk/Guide-to-diabetes/Managingyour-diabetes/15-healthcare-essentials/What-are-the-15Healthcare-Essentials/\#hce1 (2017, accessed 26 April 2017).

38. Dyer PH, Lloyd CE, Lancashire RJ, et al. Factors associated with clinic non-attendance in adults with type 1 diabetes mellitus. Diabet Med 1998; 15: 339-343.

39. Wild S, Fischbacher $\mathrm{C}$ and McKnight J. Using large diabetes databases for research. J Diabetes Sci Technol 2016; 10: 1073-1078. 